\title{
Robonaut 2 - The First Humanoid Robot in Space
}

\author{
${ }^{*}$ M.A. Diftler, ${ }^{*}$ N.A. Radford, ${ }^{*}$ J.S. Mehling, ${ }^{*}$ M.E. Abdallah, *L.B. Bridgwater, ${ }^{*}$ A.M. \\ Sanders, ${ }^{*}$ R.S. Askew ${ }^{* \star}$ D. M. Linn, ${ }^{* \star *}$ J.D. Yamokoski, ${ }^{* \star *}$ F.A. Permenter, ${ }^{* \star *}$ B.K. \\ Hargrave \\ *NASA/JSC , Houston, Texas \\ **General Motors, Warren Michigan, \\ ${ }^{* * *}$ Oceaneering Space Systems, Houston Texas
}

\begin{abstract}
NASA and General Motors have developed the second generation Robonaut, Robonaut $\mathbf{2}$ or R2, and it is scheduled to arrive on the International Space Station in late 2010 and undergo initial testing in early 2011. This state of the art, dexterous, anthropomorphic robotic torso has significant technical improvements over its predecessor making it a far more valuable tool for astronauts. Upgrades include: increased force sensing, greater range of motion, higher bandwidth and improved dexterity. R2's integrated mechatronics design results in a more compact and robust distributed control system with a faction of the wiring of the original Robonaut. Modularity is prevalent throughout the hardware and software along with innovative and layered approaches for sensing and control. The most important aspects of the Robonaut philosophy are clearly present in this latest model's ability to allow comfortable human interaction and in its design to perform significant work using the same hardware and interfaces used by people. The following describes the mechanisms, integrated electronics, control strategies and user interface that make R2 a promising addition to the Space Station and other environments where humanoid robots can assist people.
\end{abstract}


Robonaut 2 - The First Humanoid Robot in Space

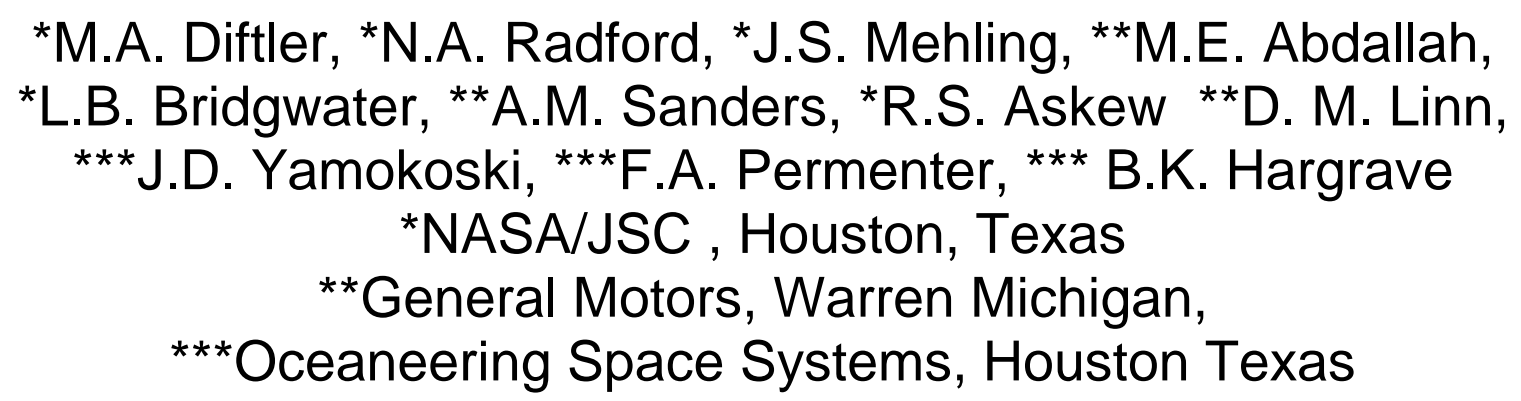

Abstract-NASA and General Motors have developed the second generation Robonaut, Robonaut 2 or R2, and it is scheduled to arrive on the International Space Station in late 2010 and undergo initial testing in early 2011 . This state of the art, dexterous, anthropomorphic robotic torso has significant technical improvements over its predecessor making it a far more valuable tool for astronauts. Upgrades include: increased force sensing, greater range of motion, higher bandwidth and improved dexterity. R2's integrated mechatronics design results in a more compact and robust distributed control system with a faction of the wiring of the original Robonaut. Modularity is prevalent throughout the hardware and software along with innovative and layered approaches for sensing and control. The most important aspects of the Robonaut philosophy are clearly present in this latest model's ability to allow comfortable human interaction and in its design to perform significant work using the same hardware and interfaces used by people. The following describes the mechanisms, integrated electronics, control strategies and user interface that make R2 a promising addition to the Space Station and other environments where humanoid robots can assist people. 


\section{INTRODUCTION}

NASA and General Motors have a history of working together, taking on formidable challenges, that date back to the Apollo Lunar Rover (need reference). The two organizations have come together again and this time to address a new challenge, developing robot assistants that can work in proximity to humans.

(Brief paragraph about how we respect Justin, Asimov, Partner robots etc, with references)

General Motors has been a leader in the application and development of robotic technology since its initial collaboration with Joseph Engelberger. GM was the first manufacturer to use industrial robots with its application of Unimate robots in 1961. Today, General Motors employs uses over 25,000 robots in its Manufacturing Operations worldwide. GM has influenced the industry over the years by leading technical development efforts in servo electric welding robots, paint application robots, the Unimate PUMA robot for light assembly, and fixturing robots.

Although performance, capability and reliability have greatly improved since the first introduction of robots in GM manufacturing, many of the targeted applications for robot use remain the same. Current industrial robots operate in a highly structured task environment and are designed and programmed to work in enclosed workcells. The consistency of the task structure enables robots to safely perform their tasks. However, this same structure also limits the robot task flexibility. While there has been some technical progress that enables robots to operate in manufacturing operations with less structure, the full technical capability is still not mature, and has not been realized. This "capability gap" has limited the range of robot applications for less structured environments.

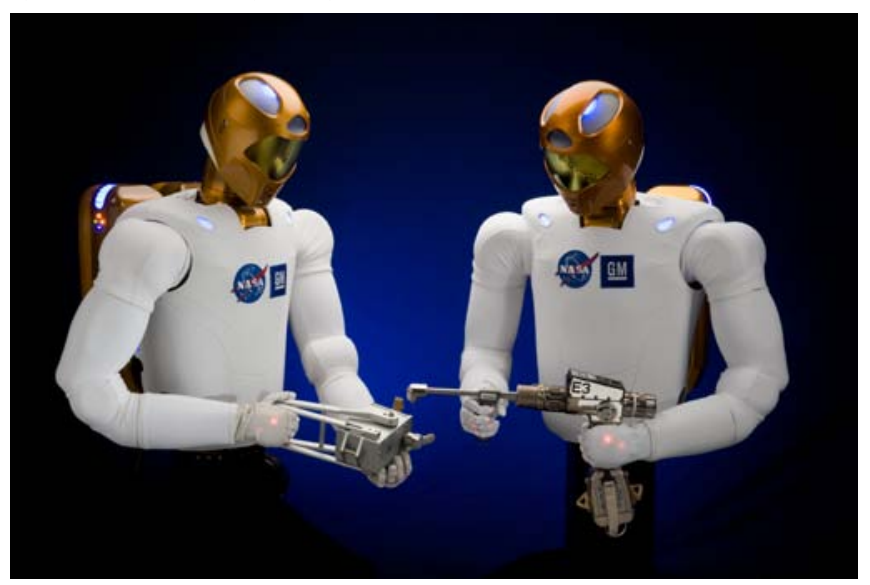

Fig. 1: Robonaut 2: units $A$ and $B$

NASA experiences a very similar robotics "capability gap." The 
challenge in this case is to make more Extra-Vehicular Activity (EVA) tasks robotically compatible. Many of the maintenance tasks on the International Space Station (ISS) are robotically serviceable and the Canadian Space Agency's Special Purpose Dexterous Manipulator (SPDM) currently on-board ISS has the capability to perform these tasks. However, to perform its job SPDM, utilizes different approach corridors than human EVA and must interface with specialized robotically compatible interfaces. While specialized worksites and interfaces for robotics system have been very successful in space they only address a portion of the servicing for ISS. Astronauts working with EVA compatible tools will perform a considerable amount of the maintenance activities.

NASA developed Robonaut 1, R1, (ref) to assist the crew in these servicing tasks and reduce this "capability gap." R1 has demonstrated, in high fidelity ground based demonstrations, its ability to work with existing EVA tools, and interfaces within the constraints of EVA worksites. Anthropomorphic robots, like R1, are well suited for applications in an unstructured environment and are fully expected to reduce the work load on EVA crew by performing routine maintenance, assisting crew members before, during, and after EVA, and serving in a rapid response capacity.

NASA's success in developing and demonstrating R1's capabilities attracted the attention of GM. GM approached NASA in 2006 as part of a worldwide review of humanoid robotics in search of new technologies that would help their skilled workforce improve product quality and manufacturing assembly processes. A detailed review of the Robonaut 1 system convinced GM that NASA's expertise in upper body systems specifically designed to assist astronauts made the agency an excellent partner for developing the robotic technologies that would also meet GM's goals of closing the "capabilities gap". It was realized by both organizations that there were enormous benefits from a robust anthropomorphic robotic system that relieves people factory workers or astronauts from dangerous or ergonomically painful and difficult activities. 
For NASA and GM to achieve the desired improvements, Robonaut 2 required a number of significant advancements in the robot's electromechanical design, sensing integration, controls strategy, and user interface. At the heart of these advancements are technologies and approaches that allow for increased speed, strength, and dexterity while not sacrificing, and in fact improving upon, a system design compatible with direct human interaction that hads always been a focus of previous Robonaut development. (arkward)

\section{MECHATRONIC DESIGN FOR RELIABILITY}

With 42 independent degrees of freedom, 50 motors and over 350 sensors, R2, shown in figure 1 , is a mechatronic integration challenge. Prior lab experience on R1 and other robots demonstrated a direct correlation between the overall wire count in a robotic system and the reliability of said system. R1 had a centralized processing and point to point communication paradigm which necessitated large conductor count cabling and long runs of sensitive analog signals. Therefore, the avionic architecture for R2 was designed and developed around one central theme - the reduction of the conductor count in the robot and specifically the two arms.

In order to fundamentally reduce the conductor count, a new communication scheme was required. R1 was built upon a point to point RS-485 communication architecture that, including power and other relevant signals, required over 100 conductors in each main arm cable. This produced an unwieldy amount of wires that often got damaged during unrelated servicing and the wires themselves were often the reason for other maintenance required on the robot. Moreover, that amount of wiring for each arm forced the cabling to reside externally whereby it created additional challenges in how to manage to service loop. In contrast, R2 was designed to have a distributed processing architecture with a high speed serial communication network that utilized a bussed power configuration. This afforded the robot a minimal set of wires for each internal main arm cable 
which totaled only 16 . The high scale, 5 degree-of-freedom upper speed serial communication arms. The use of series elastic structure is a custom protocol actuation, however, differentiates which utilizes Multi-drop Low R2 from previous designs. Voltage Differential Signal Developed initially with legged (MLVDS) as the physical layer robots in mind, series elastic with bus speeds of 50Mbit. Each actuators (SEA's) have been node on the MLVDS network shown to provide improved shock makes up the vital string of tolerance, beneficial energy controllers that provide the low storage capacity, and a means for level joint control and data accurate and stable force control processing. The upper arm has 5 [1,2]. Not surprisingly, these nodes and the lower arm consists features are of interest to the of two additional nodes; one for manipulation community as well the forearm and one for the hand.

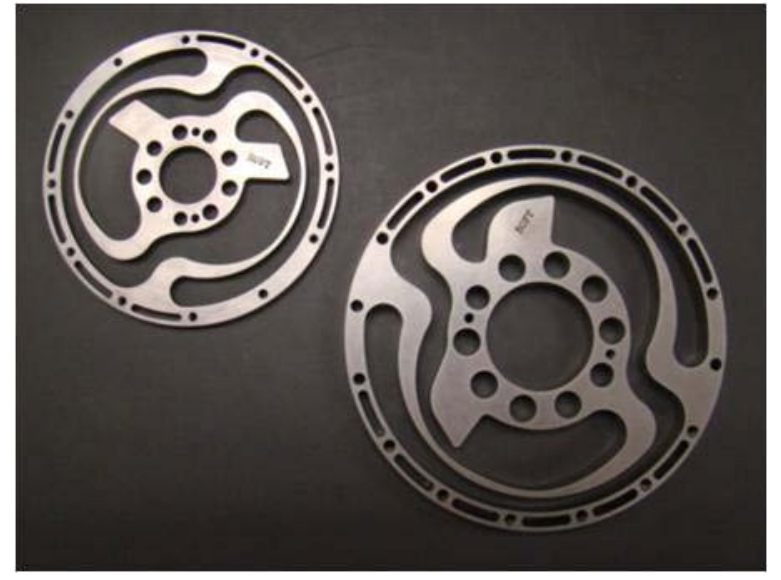

Fig. 2: A picture of multiple sizes of arm spring

\section{A. Upper Arms}

Robonaut 2, like its predecessor, uses brushless DC motors, harmonic drive gear reductions, and electromagnetic failsafe brakes as the building blocks for the power and torque dense actuators in the robot's human- and a number of humanoid robot arms have been designed with passive compliance between their joints' actuators and outputs [citation needed]. Robonaut 2 is unique among these robots, in that it does not sacrifice strength, or payload capacity, to achieve fine torque sensing at each of its joints. This is made possible by the custom planar torsion springs that are integrated into each arm actuator and the two 19 bit absolute angular position sensors that measure each spring's deflection. Shown in Fig. 2, these springs are uniquely sized for each arm joint and are capable of elastic deformation across the full range of their actuators' continuous torques. This gives 
Robonaut 2 the fine force resolution to implement a variety of impedance control modes at both the joint and Cartesian level while still enabling the robot to handle significant human-scale payloads as in Fig. 3.

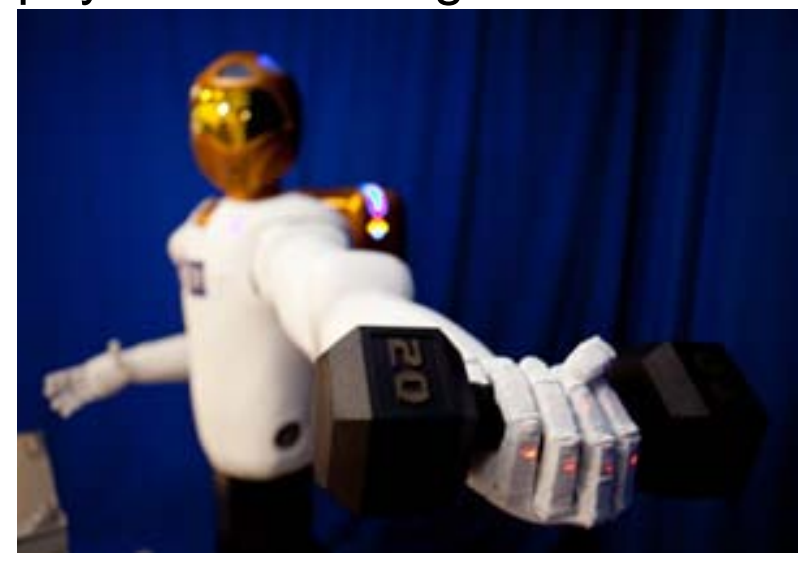

Fig. 3: A picture of R2 weight lifting (clearly seeing the 20lb)

In order to fully realize the upper arm capability, a considerable amount of capability had to be placed on the low level joint controllers. In R1, the joint loops were closed at a high level central processor and this fundamentally limited the joint loop rates and the type of sensors used in that control. Therefore, each upper arm node consists of a highly integrated joint controller, called a Superdriver, which serves as the workhorse of the upper arm motion control strategy. The Superdriver is an FGPA based controller with an embedded
PowerPC

processor

and

programmable logic coupled with a 3 phase brushless DC inverter bridge. This Superdriver performs motor commutation and current control,

serialization/deserialization of joint data and commands, sensor processing, and control of the SEA's noted above. The embedded processor run $10 \mathrm{KHz}$ sensor processing, current and SEA control

loops. Programmable logic is used for the MLVDS and motor commutation, which can either be six-step or space vector. SEA control can be configured locally for joint torque or joint impedance.

The Superdriver provides a streamlined means of interfacing to the analog and digital joint sensors. These sensors need only communicate with a local processor which is responsible for all the joint control. Sensors read at each joint include three motor phase currents, motor bus current, temperature sensors, two absolute position sensors (APS), and one incremental motor encoder. The motor current sensors are used for vector control and torque limiting. The absolute position sensors are 
used in tandem to measure SEA spring deflection. One APS is used for joint position estimation. The incremental motor encoder is used for vector commutation and also gives a redundant measurement of input SEA spring position if harmonic drive dynamics are ignored. Finally, the output position is differentiated at the joint to provide an estimate of joint velocity.

One of the main achievements of the Superdriver design is how highly integrated the printed circuit board is into the overall upper arm joint design. Each Superdriver board plugs in to each joint with 2 blind mate connectors. This provides a clean and robust interface for each board and places zero stress on any related wiring which prevents life and fatigue related failures. Each circuit board is also very accessible makinges routine maintenance and repairs easy.

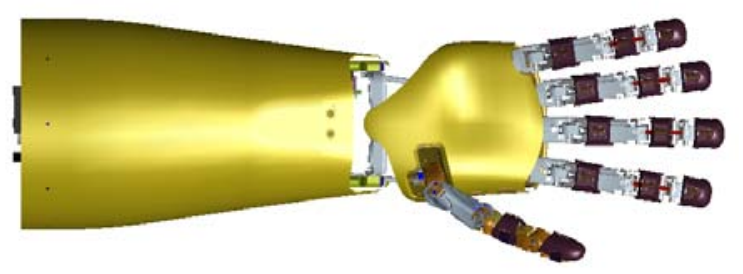

Fig. 4: Robonaut Hand (reat picture?)Design

\section{B. Hands}

Robonaut 2's hand, shown in Figure 4 , is designed to more closely approximate human hand capabilities than its predecessor, Robonaut (Lovchik paper reference). With a two-DOF wrist, and a 12-DOF hand with five fingers, the forearm houses 18 motors, eight separate circuit boards. The forelimb is selfcontained, requiring only power and communication. Robonaut 2's hand has demonstrated its capabilities to use a large set of EVA tools, conventional hand tools, and soft goods.

The hand, shown in figure 5 , is divided into a dexterous set used for manipulation and a grasping set used to maintain a stable grasp while working with large tools. The dexterous set consists of two three-DOF fingers, the index and middle finger, and a four-DOF opposable thumb. The grasping set consists of two oneDOF fingers, the ring and little finger. All fingers are shockmounted within the palm, giving the hand rugged grasping options. 
Dexterous set

Grasping set

Fig. 5: R2 Finger groupings

The quality of the Robonaut 2 hand was determined by its ability to emulate Cutkosky's grasp taxonomy[ref]. The original Robonaut hand was only able to successfully emulate $\sim 50 \%$ of the grasps, while Robonaut 2 has the ability to emulate $90 \%$ of the grasps. Figure 6 shows the Robonaut 2 hand in the Cutkosky's grasps. The increase in capability of the hand came about by advancements made in the thumb design. The Robonaut 2 thumb has four DOFs, is the same scale, and closely approximates the kinematic layout of a human thumb. In addition to the kinematic layout, the thumb actuation scheme is designed such that it is significantly stronger than the fingers that it is in opposition to.

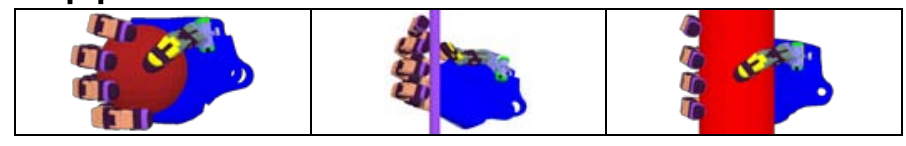

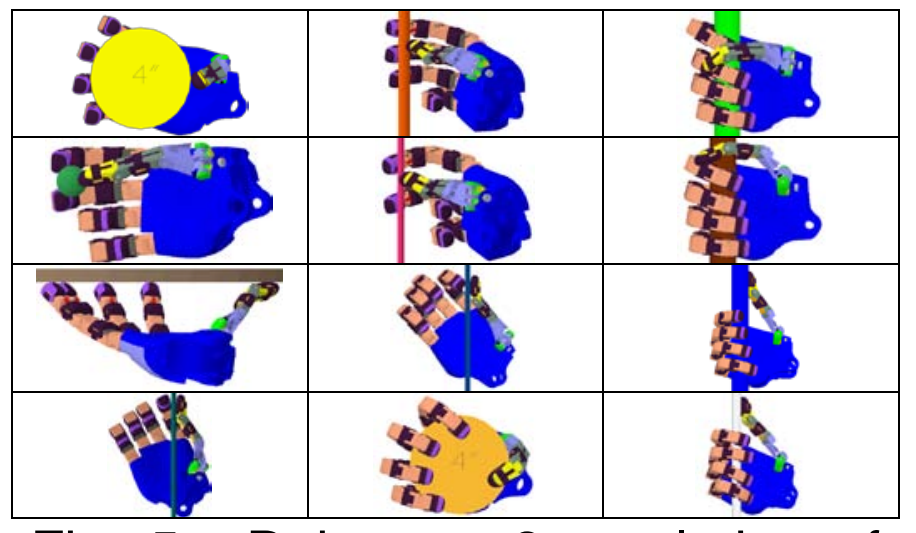

Fig. 5: Robonaut 2 emulation of Cutkosky's Grasp Taxonomy

Each digit is controlled by a set of linear actuators that are extrinsically located in the forearm. An N+1 configuration [Salisbury ref] is implemented and allows for a minimal set of actuators for each DOF. Each finger phalange has the ability to contain a six-DOF load cell (ref patent number $7,784,363$ ) for a total of 14 load cells. The sensors provide all three axes of force and moment, allowing for measurement of external contact forces as well as shear force and slippage of objects held by Robonaut 2. Additional sensors are embedded within the palm providing tendon tension measurement. The tendon tension sensors measure the internal forces of the antagonistic actuation of each finger.

The forearm contains all the 
power/data distribution and processing electronics for the finger and wrist actuators. Only 6 wires pass from the upper arm to the lower arm. There are 2 different types of electronic control boards in the forearm. At the top level is the forearm controller and below that is the forearm driver electronics. A single forearm controller board serves as a supervisor/ controller for the forearm driver electronics and communicates via MLVDS with the robot's central IO processor. There are 6 motor controller boards, each of which interface to and drive 3 finger or wrist motors. In total the motor controller boards command 16 finger motors and 2 wrist motors.

The forearm motor driver boards have phase current sensing and provide the ability to set a continuous current limit, as well as allow a brief current override in case a pulse of current is required for a short amount of time. A separate over-current bit will trigger if the max current threshold is exceeded. This triggers a fault in the driver which can be reset at the brainstem level once an evaluation is performed.

\section{CONTROl StRATEgy}

\section{A. Implementation}

The control software for R2 is a multi-threaded application spread across two PowerPC processor cards in a Compact $\mathrm{PCl}$ (cPCl) chassis. The application code was developed in ControlShell ${ }^{R}$ and runs on the $V x W^{2}$ rks $^{\mathrm{R}}$ operating system. The first processor handles all the collection and high-level processing of the sensor information from the embedded network. It also oversees an extensive safety system evaluating the kinematics and force levels in the system. The second processor implements the control law and computes the kinematics. Sensor data and computed quantities are shared between the two processors via a shared memory region through the PCI backplane.

These central processors pass down desired torque commands to the motor drivers. The embedded processors there implement a high speed torque control loop running at $10 \mathrm{kHz}$.

\section{B. Architecture}

The controls architecture was designed to provide an impedance based control system with great 
flexibility. The concept of PID force control objective can impedance control provides for preempt the primary operational robust interaction with the space relation to create a mixed environment, while allowing for force/impedance control behavior both motion and force control in operational space.

objectives (Hogan 1987). The The secondary joint-space architecture was designed to allow impedance relation then governs for multiple, prioritized tasks that the joint motion lying in the nullcan control the robot with respect space of the primary task, to different spaces and different including the joints downstream of nodes of interest in the kinematic the selected node. When no node tree. At the heart of the framework is an impedance law with two hierarchical relations: an operational-space impedance at top priority, and a joint-space impedance at second priority. Multiple such laws can be implemented hierarchically.

The primary operational space relation can be defined with respect to any of a number of possible nodes in the tree, and it can be defined with respect to either the linear and/or the angular motion. Given the task definition, the relevant kinematics are generated in real-time for all joints upstream of the selected node in the kinematic chain. The waist joint can be optionally included in the kinematic chain, allowing the arms to be controlled either independently or integrated for whole-body motion. In addition, a on a respective arm is selected for the primary task, this joint-space relation fully governs all joints of the arm.

C. Arms Control Law

The dual-priority control described in the previous subsection is defined as follows. First, consider the equations of motion for the full system of manipulators.

$M \ddot{q}+c+g-\tau_{e}=\tau$

$M$ is the joint-space inertia matrix. $q$ is the column matrix of joint angles. $c$ is the column matrix of Coriolis and centripetal generalized forces. $g$ is the column matrix of gravitational generalized forces. And $\mathrm{T}$ and $\mathrm{T}_{\mathrm{e}}$ are the column matrices of actuated and external torques, 
respectively.

Second, consider the desired closed-loop impedance relations for both the operational and joint spaces.

$M_{o} \ddot{x}+B_{o} \dot{x}+K_{o} \Delta x=F_{e}$

$M_{j} \ddot{q}+B_{j} \dot{q}+K_{j} \Delta q=\tau_{e}$

$M_{o}, B_{o}$, and $K_{o}$ represent the desired operational-space inertia, damping, and stiffness matrices, respectively. $M_{j}, B_{j}$, and $K_{j}$ represent the desired joint-space inertia, damping, and stiffness matrices, respectively. $x$ and $F_{e}$ represents the operational-space coordinates and corresponding external forces, respectively. The $\Delta$ indicates the error in the respective variable with respect to its desired value.

To eliminate the need for sensing of the external torques, the impedance inertias are set to the passive inertia of the system: $M_{0}=M, M_{j}^{-1}=J M^{-1} J^{T}$. The full solution for this dual-priority impedance control law is presented in (Platt 2010). For the sake of the implementation, the following approximation was employed to eliminate the need for the inertia matrix.
$\tau=-J^{T}\left(B_{o} \dot{x}+K_{o} \Delta x\right)-N\left(B_{j} \dot{q}+K_{j} \Delta q\right)+g$

$N=I-J^{+} J$

$\mathrm{J}$ is the Jacobian matrix mapping joint velocities to operationalspace velocities, and $N$ is the nullspace projection matrix for $J$. A closed-loop analysis shows that this control law provides the desired joint-space impedance relation (2) projected into the nullspace. In the range-space, it provides the desired operationalspace impedance relation (2) with a disturbance from the null-space dynamics. The effects of this disturbance, as well as the effects of neglecting the Coriolis forces and the derivative of $\mathrm{J}$, are negligible at the speeds R2 operates at.

A similar relationship to (3) is used for mixed force/impedance mode. A null-space projection matrix for the force task Jacobian is used to project the primary and secondary tasks into the force task's null-space.

\section{Fingers Control Law}

Since the fingers are actuated by coupled tendons, rather than independent drives, a special control law is needed for them. It turns out that the state-of-the art in force control of tendon-driven 
manipulators actually controlled the manipulators in the tendonspace, which exhibit a first-order coupled disturbance between the joints. Alternatively, the control law can be formulated in the jointspace to eliminate this coupled disturbance and increase the speed of the response. A full discussion of this control law is available in (Abdallah 2010). We present here the final control law, where an inner position control loop is implemented on the actuators.

$$
p_{c}=p-k_{d} \dot{p}+\left[\begin{array}{c}
R \\
W
\end{array}\right]^{T} K_{p} \Delta\left(\begin{array}{l}
\tau \\
t
\end{array}\right)
$$

$p$ and $p_{c}$ represent the actual and commanded positions for the actuators. $R(n \times n+1)$ represents the kinematic mapping from tendon tensions to joint torques. $W$ is a row matrix selected orthogonal to $R$. $t$ is a scalar measure of the internal tension on the tendon network, defined as $t=$ Wf. $K_{p}$ and $k_{d}$ are constant gains.

IV. WORKING IN PROXIMITY TO

$$
\text { HUMANS }
$$

Both industrial manipulators and R1 utilize kill switches or emergency stop buttons as part of their standard safety systems. In the case of industrial manipulator, additional safety devices such as light curtains or sensor mats also provide power cutoffs in the event of a human entering the robots workspace. R2 has moved away from this paradigm. The basic torque control strategy noted above limits the force the robot applies to the environment ensuring that when inadvertent contact does occur, the resulting force felt by the human is comfortable and the robot can be manually restricted by pushing on a limb.

In parallel to the basic torque control are software monitoring routines that use the torque sensing at the arm and waist joints and multiple force sensors in the arms, to independently monitor the robot's forces. If a predefined limit is exceeded at either the joint or the arm level, the robot disengages motor power and stops. A multitude of additional software routines on several processors continuously check the health and communication of the two main force/torque monitoring loops. The result is a triple redundant 
system to keep forces within allowable limits.

The benefit of this architecture is that the R2 on the ISS will not need an Emergency Stop button. This E-stop will be available and is now a Motion-Stop or M-stop. The robot operator has the discretion to use the M-stop as a convenience while using the robot, but crew will be allowed into the robot's workspace without having to use it.

\section{USER INTERFACE}

One of the unique challenges of working with R2 is distilling the control of a complicated system into a user-friendly design. In industrial and commercial environments, suppliers have created interfaces to move, monitor, and manage standard robotic arms. Unfortunately, no commercial supplier offers a standard interface to control a 42 degree of freedom humanoid robot. (expand to include teleop?)

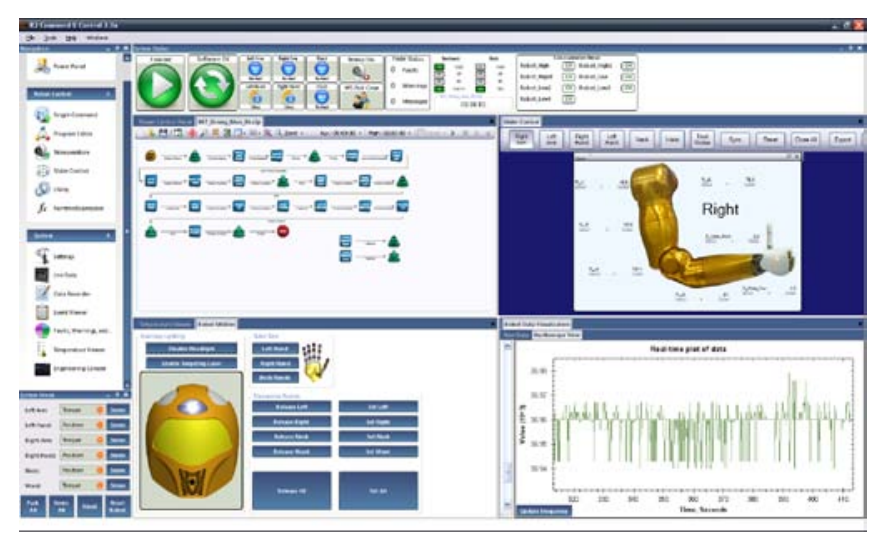

The custom R2 interface uses common elements such as buttons and sliders to provide the user with direct control over the basic functionality of the robot. These objects are organized by function and presented to the user based on the pre-determined needs of a variety of tasks. This simple interface enables novice users to perform basic tasks on the robot without requiring excessive training.

The interface uses a monitored network link to receive telemetry, events, and status updates from the system and to send commands. The data is displayed on the screen using information rich symbols and controls. The user interface modifies the visual appearance of these objects to highlight conditions that require additional attention. These objects and features enable the operator to filter the large amount 
of available data and more easily understand the state of the robot. The interface also provides the ability to log and graph both incoming and outgoing data.

The interface is dynamically configurable by the user. This allows the operator to move the displays and controls to an orientation that is well suited for the current task.

In addition to the basic control of the robot using regular input methods, the user interface also provides a custom program language. This language is designed to work closely with the design methodologies implemented in the robot hardware and controls algorithms. The language provides a flexible way to integrate sequenced motion with the myriad of sensor platforms available to R2.

\section{Activities IN SPACE}

The Robonaut 2 robot will be the first humanoid type robot sent to space. The robot will deployed on the International Space Station, and will be used to perform a variety of different experimental tasks. While working side-by-side with its human astronauts, the R2 robot will be tested for its ability to actuate switches, use standard tools, and manipulate softgood objects and cables. The results of these experiments will help develop algorithms and control systems with even more capability.

\section{CONCLUSIONS}

Robonaut 2 is a significant advancement in dexterous robotics. Not only will it be a first humanoid robot is space it represents a significant advance over its predecessor in terms of strength, speed and dexterity. The...

\section{ACKNOWLEDGMENT}

The authors would like to thank the entire Robonaut 2 team for their tireless dedication to excellence: The team includes the authors and the following individuals: Chris Ihrke (General Motors), Don Davis (General Motors), Philip Strawser (NASA), Adam Parson (OSS), Charles Wampler (General Motors), Roland Menassa(General Motors), Matt Reiland (General Motors)... (more to comeadded)

\section{REFERENCES}

[1]Bluethmann, et_al_t Robonaut Astronaut Assistant. 
[2]Variety of other humanoid papers - due diligence

[3]Pratt, G. A. and Williamson, M.

M. "Series Elastic Actuators."

in proceedings of IEEE/RSJ International Conference on Intelligent Robots and Systems (IROS 95). Pittsburgh, PA. 1995. pp. 399-406.

[4]Robinson, D. W. et al. "Series Elastic Actuator Development for a Biomimetic Walking Robot." in proceedings of IEEE/ASME International Conference on Advanced Intelligent Mechatronics. 1999. pp. 561-568

[5]Lovchik, C, Diftler, M. ICRA Robonaut paper 1999.

[6]M. R. Cutkosky: "On Grasp Choice, Grasp Models, and the Design

of Hands for Manufacturing Tasks", IEEE Transactions on Robotics and Automation, Vol. 5, No. 3, pp. 269-279, 1989.

[7]N. Hogan, "Impedance control: An approach to manipulation IIII," Journal of Dynamic Systems, Measurement, and Control, vol. 107, pp. 1-24, 1987.

[8]M. Abdallah, R. Platt, C. Wampler, B. Hargrave, "Applied Joint-Space Torque and Stiffness Control of Tendon-
Driven Fingers," IEEE-RAS Intl. Conf. on Humanoid Robots, Nashville, TN 2010.

[9]R. Platt, M. Abdallah, C. Wampler, "Multiple-priority impedance control," in submission to IEEE Intl. Conf. on Robotics and Automation, 2010. 
${ }^{4359847}$ nnnm 